\title{
Erratum: Dynamic process and Uhlmann process: Incompatibility and dynamic phase of mixed quantum states [Phys. Rev. B 101, 104310 (2020)]
}

\author{
Hao Guo, Xu-Yang Hou, Yan He, and Chih-Chun Chien $(\mathbb{0}$
}

(Received 26 September 2020; published 14 October 2020)

DOI: 10.1103/PhysRevB.102.139901

In our paper, the time-ordered integral of the dynamic phase shown in Eq. (49) is not treated correctly. In the following, we present the correct results for the Su-Schrieffer-Heeger (SSH) and Kitaev models. Importantly, the conclusions remain qualitatively the same.

We consider the generic one-dimensional quadratic Hamiltonian shown in Eq. (47) of the original paper with the density matrix of each $k$ given by Eq. (48) of our paper. The total density matrix is then $\rho=\prod_{k} \otimes \rho_{k}$, where $k$ is from the first Brillouin zone. A full treatment of the time-ordered integral shown in Eq. (49) of the original paper leads to unmanageable numerical evaluations. Instead, we consider the situation where the Hamiltonian is time independent when the system evolves. Thus, the time-ordered integral can be evaluated exactly. We obtain

$$
\begin{aligned}
\theta_{D} & =\arg \operatorname{Tr}\left[\rho \exp \left(-\frac{i}{\hbar} \int_{0}^{\tau} H d t\right)\right] \\
& =\arg \prod_{k} \operatorname{Tr}\left[\rho_{k} \exp \left(-\frac{i}{\hbar} H_{k} \tau\right)\right] \\
& =\sum_{k} \theta_{D}(k) \bmod 2 \pi,
\end{aligned}
$$

where $\tau$ is the duration of the time evolution, $\theta_{D}(k)=\arg \operatorname{Tr}\left[\rho_{k} \exp \left(-\frac{i}{\hbar} H_{k} \tau\right)\right] . \theta_{D}$ is the sum of the dynamic phase with different values of $k$.

For the two-level system given by Eq. (47) of our paper, it can be shown that

$$
e^{-(i / \hbar) H_{k} \tau}=e^{-(i / \hbar) f_{k} \tau}\left[\cos \left(\omega_{k} \tau\right) 1_{2 \times 2}-i \sin \left(\omega_{k} \tau\right) \hat{\mathbf{n}}_{k} \cdot \vec{\sigma}\right] .
$$

where $\omega_{k}=\frac{\Delta_{k}}{2 \hbar}$. After some algebra, we get

$$
\theta_{D}=\sum_{k}\left\{\arg \left[\cos \left(\omega_{k} \tau\right)+i \sin \left(\omega_{k} \tau\right) \tanh \left(\frac{\beta \Delta_{k}}{2}\right)\right]-\frac{f_{k} \tau}{\hbar}\right\} \bmod 2 \pi
$$

In the continuum limit, $\sum_{k} \rightarrow \frac{1}{2 \pi \hbar} \int_{0}^{2 \pi} d k$.

For the periodic Kitaev chain described by Eq. (50) of the original paper. $\Delta_{k}=2 M \sqrt{(c \cos k-m)^{2}+\sin ^{2} k}$ and $\hat{\mathbf{n}}_{k}=$ $\frac{2 M}{\Delta_{k}}(0,-\sin k,-m+c \cos k)^{T}$. The dynamic phase of $k$ is given by

$$
\begin{aligned}
\theta_{D}(k)= & \arg \left[\cos \left(\omega_{k} \tau\right)+i \sin \left(\omega_{k} \tau\right) \tanh \left(\frac{\beta \Delta_{k}}{2}\right)\right] \\
& = \begin{cases}\arctan \left[\tan \left(\omega_{k} \tau\right) \tanh \left(\frac{\beta \Delta_{k}}{2}\right)\right], & \text { if } \omega_{k} \tau \in\left(2 n \pi-\frac{\pi}{2}, 2 n \pi+\frac{\pi}{2}\right), \\
\pm \frac{\pi}{2}, & \text { if } \omega_{k} \tau=2 n \pi \pm \frac{\pi}{2}, \\
\arctan \left[\tan \left(\omega_{k} \tau\right) \tanh \left(\frac{\beta \Delta_{k}}{2}\right)\right]+\pi, & \text { if } \omega_{k} \tau \in\left(2 n \pi+\frac{\pi}{2}, 2 n \pi+\pi\right), \\
\arctan \left[\tan \left(\omega_{k} \tau\right) \tanh \left(\frac{\beta \Delta_{k}}{2}\right)\right]-\pi, & \text { if } \omega_{k} \tau \in\left(2 n \pi-\pi, 2 n \pi-\frac{\pi}{2}\right) .\end{cases}
\end{aligned}
$$

We also consider the periodic SSH model described by Eq. (54) of the original paper. The dynamic phase of $k, \theta_{D}(k)$, is also given by Eq. (4) but with $\Delta_{k}=2 \sqrt{J_{1}^{2}+J_{2}^{2}+2 J_{1} J_{2} \cos k}$ and $\hat{\mathbf{n}}_{k}=\frac{2}{\Delta_{k}}\left(-J_{1}-J_{2} \cos k, J_{2} \sin k, 0\right)^{T}$.

Figure 1 shows the total dynamic phase as a function of the time duration of the dynamic process for selected temperatures. The upper and lower panels are for the Kitaev chain and SSH model, respectively. There are periodic sharp peaks coming from the combined effects from the summation of dynamic phases with different $k$ 's and the modulo operation of $2 \pi$ on the phase. In Fig. 2, we analyze the behavior of $\theta_{D}(k)$ with a fixed value of $k$ by plotting $\theta_{D}(k)$ vs $\tau$ with $k=\frac{\pi}{3}$ at selected temperatures. 

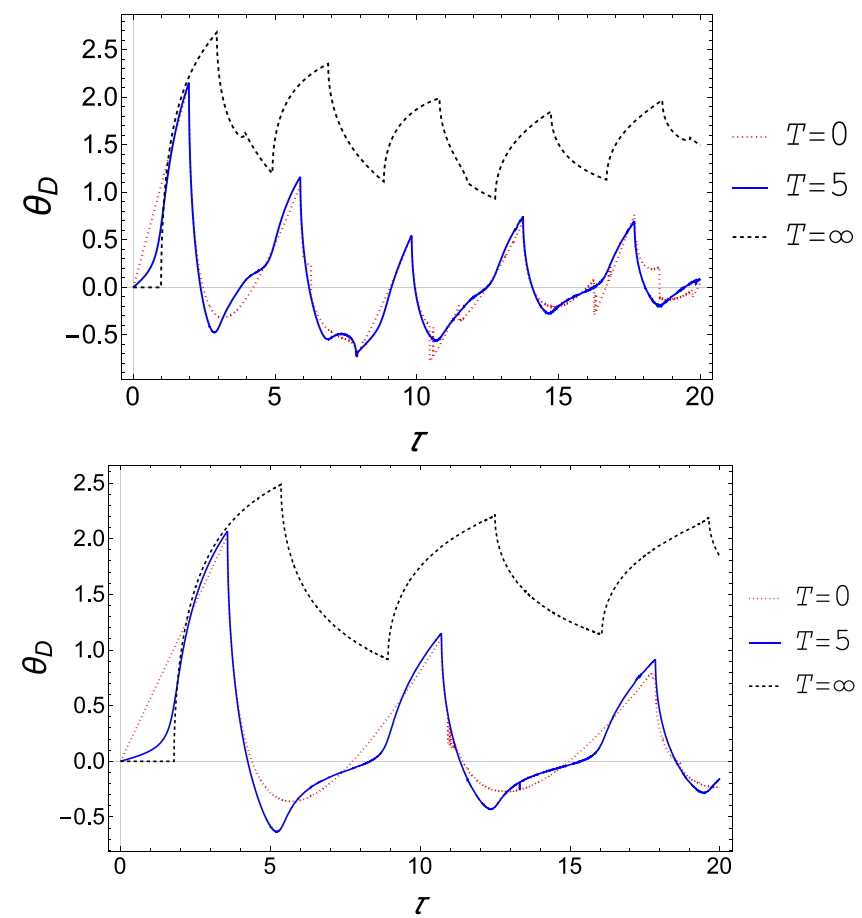

FIG. 1. The total dynamic phase of the Kitaev model (upper panel) and the Su-Schrieffer-Heeger (SSH) model (lower panel) as a function of $\tau$ (in units of $\hbar / M$ and $\hbar / J_{1}$ ). The red dotted lines, blue solid lines, and black dashed lines correspond to $T=0,5$, and $\infty$ (in units of $M / k_{B}$ and $J_{1} / k_{B}$ ), respectively. Here, $c=1.0$ and $m=0.6$ for the Kitaev model and $J_{1}=0.4$ and $J_{2}=0.48$ for the SSH model.
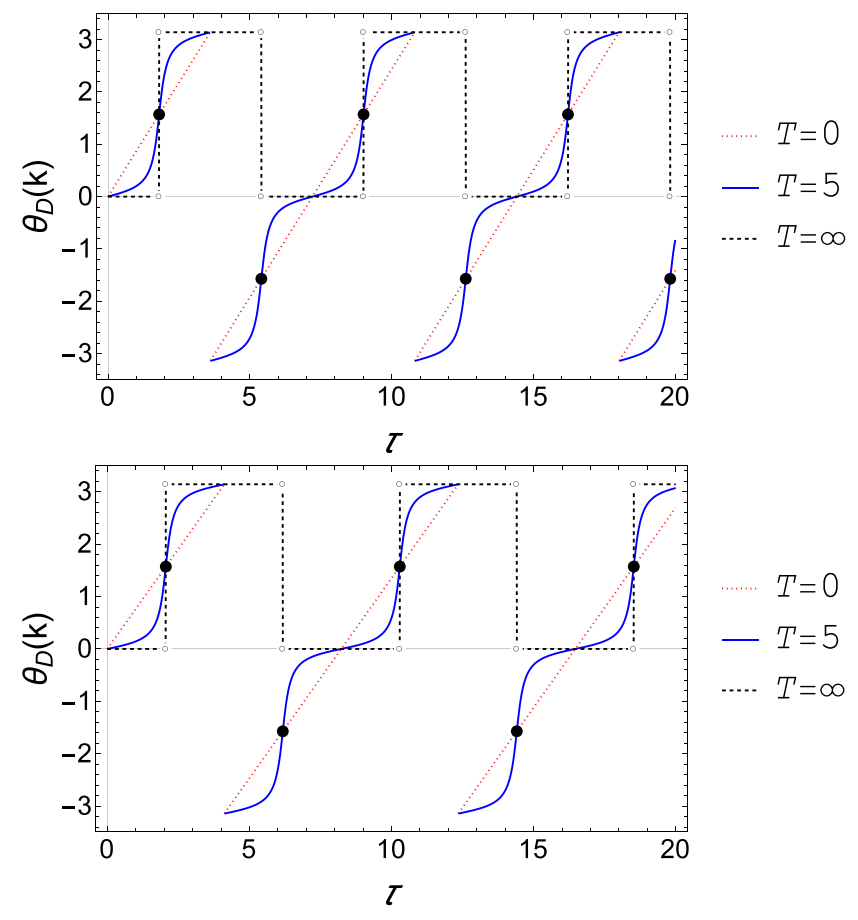

FIG. 2. The dynamic phase with $k=\frac{\pi}{3}$ for the Kitaev model (upper panel) and the SSH model (lower panel) as a function of $\tau$ (in units of $\hbar / M$ and $\hbar / J_{1}$ ). The red dotted lines, blue solid lines, and black dashed lines correspond to $T=0,5$, and $\infty$ (in units of $M / k_{B}$ and $J_{1} / k_{B}$ ), respectively. The black dots indicate the value of $\theta_{D}\left(\frac{\pi}{3}\right)$ at $T=\infty$ and $\tau=\frac{2 n \pi \pm \frac{\pi}{2}}{\omega_{\pi / 3}}$, where $n$ is an integer. The parameters are the same as those in Fig. 1. Since $\omega_{\pi / 3}$ of the two models are different, the periods of $\theta_{D}\left(\frac{\pi}{3}\right)$ are also different. 
The fixed- $k$ dynamic phase exhibits similar behavior as the results shown in our paper. Take the Kitaev chain as an example, $\theta_{D}\left(\frac{\pi}{3}\right)=\omega_{\pi / 3} \tau(\bmod 2 \pi)$ shows a linear dependence at zero temperature according to Eq. (4). At finite temperatures, $\theta_{D}\left(\frac{\pi}{3}\right)$ is a periodic and smooth function of $\tau$. At infinite temperature, $\theta_{D}\left(\frac{\pi}{3}\right)$ takes discrete values. The regime $\omega_{\pi / 3} \tau \in\left(\frac{\pi}{2}, \frac{3 \pi}{2}\right) \bmod 2 \pi$ are the resonant points because $\theta_{D}\left(\frac{\pi}{3}\right)=\pi$ at infinite temperature. The ordinary points in $\theta_{D}\left(\frac{\pi}{3}\right)=\pi$ have $\theta_{D}\left(\frac{\pi}{3}\right)=0$ at infinite temperature according to Eq. (4). At the points $\omega_{\pi / 3} \tau= \pm \frac{\pi}{2} \bmod 2 \pi, \theta_{D}\left(\frac{\pi}{3}\right)= \pm \frac{\pi}{2}$ independent of $T$. Therefore, the dynamic phase with multiple discrete values in cyclic quasistatic processes at infinite temperatures exist in $\theta_{D}(k)$ for each $k$ value. The total dynamic phase, however, exhibits more complicated behavior. The results of the SSH model are qualitatively similar, although the different expression of $\omega_{k}$ leads to a different period of $\theta_{D}(k)$ when compared to the Kitaev model. 